\title{
Expansion the Output Swing of the Ultra-Low Noise Transimpedance Amplifier for Microcurrent Measurement
}

\author{
Xueyou Shi ${ }^{a}$, Kai Xie ${ }^{b}$, Bin Sun ${ }^{c}$, Kai Zhao ${ }^{d}$, Anfeng Huange \\ Xidian University, Xi'an 710126 China \\ ashixueyou2010@163.com, bkaixie@mail.xidian.edu.cn, csunbin@stu.xidian.edu.cn, \\ dzkai385@163.com, eanfenghuang@stu.xidian.edu.cn
}

Keywords: microcurrent, low noise amplifier, output swing.

\begin{abstract}
Accurate measuring of micro currents in fA range requires an ultra-low bias amplifier which has relative low output swing, limits the value of feedback resistor, and ultimately results a poor noise performances. In this paper, an improved low-noise transimpedance amplifier with a large feedback loop is proposed, so that high-value resistor can be employed to achieve low noise performance. The noise theory, operational principle and circuit implementation are discussed. The prototype is designed with measurement range of $20 \mathrm{~V} / 200 \mathrm{pA}$, the test results indicates a low white noise level of $0.5 \mathrm{fA} / \sqrt{ } \mathrm{Hz}$, and a offset bias of $\sim 2.5 \mathrm{fA}$, while providing an output voltage swing expand to above $\pm 20 \mathrm{~V}$.
\end{abstract}

\section{Introduction}

Accurate measurement of fA-pA-scale currents have been widely used in many fields, such as weather monitoring[1], electron microscopy[2], electrochemical measurement[3], bioelectricity[3,4], and ion detecting[5]. In general, a low-noise transimpedance amplifier is used as the input stage to convert and amplify the ultra-low current to a readable voltage range[4].

To obtain high measuring accuracy in microcurrent measurement, the input bias current of the input stage transimpedance amplifier should be as low as possible. Until now, the operational amplifier LMP7721 is the industry's lowest specified input bias current electrostatic amplifier, with typical values of $3 \mathrm{fA}$ input bias current. It is ideal for microcurrent measurement, but its output voltage swing is no more than $\pm 2.5 \mathrm{~V}$, which limit the current measurement range, in other words, limit the value of feedback resistor under the same range of current.

As we know, A high-value feedback resistor is a critical component of the transimpedance amplifier, because both noise characteristics and amplification gain sensitivity are determined by it. According to the Johnson noise theory, increasing feedback resistance not only improves sensitivity but also reduces the equivalent noise current. Therefore, a goal in low-noise microcurrent amplifier design is to increase feedback resistance as much as possible under the amplifier's permitted dynamic range. As a result, expanding the amplifier's dynamic range will allow the the application of high value feedback resistor, and help to achieve low noise performance.

In this manuscript, an output swing boost circuit will be presented for the transimpedance amplifier in microcurrent measurement, enable the use of high value feedback resistor, so that both high sensitivity and ultra-low-noise performance can be achieved.

The rest of this paper is organized as follows. In section 2, discussed the low noise performance of the classical transimpedance amplifier. Section 3 presents the output voltage boost circuit. Test of the output voltage and noise performance are shown in Section 4. Conclusion appears in Section 5.

\section{Noise analysis of classical transimpedance amplifier}

The typical application of transimpedance amplifier in microcurrent measurement is shown in Fig. 1(a). The first stage is a current-to-voltage converter and the second stage is an inverting amplifier that corrects the polarity and provides extra gain to expand the output voltage swing. The current conversion gain is $U_{\text {out } 1} / I_{\text {in }}=R_{\mathrm{f} 1} R_{2} / R_{1}$. 


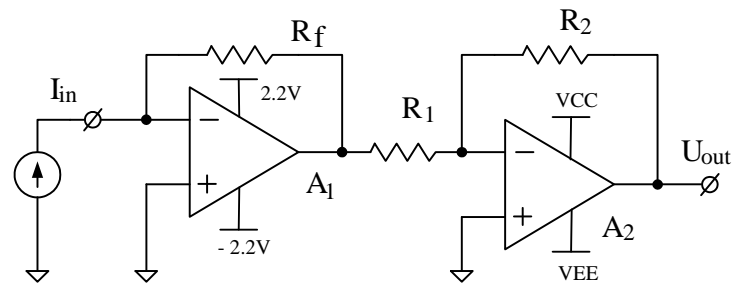

(a)

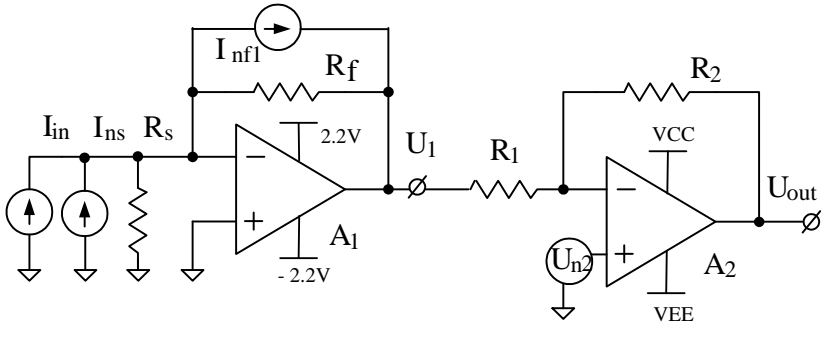

(b)

Figure 1. Typical transimpedance amplifier (a) and its noise equivalent circuit (b).

The noise equivalent circuit is illustrated in Fig. 1(b): $R_{\mathrm{s}}$ is the source resistance, $I_{\mathrm{ns}}$ and $I_{\mathrm{nf} 1}$ are the equivalent Johnson noise sources of $R_{\mathrm{s}}$ and $R_{\mathrm{f} 1}$, and $U_{\mathrm{n} 2}$ is the noise voltage contributed by amplifier A2, respectively. In this circuit, the first stage internal noise is mainly due to the Johnson noise made by resistor $R_{\mathrm{f} 1}$, expressed as $V_{n f}=\sqrt{4 k T B R_{f 1}}$. This noise, with reference to the input, is equivalent to a noise current:

$$
I_{n f 1}=V_{n f} / R_{f 1}=\sqrt{4 k T B / R_{f 1}} .
$$

The first-stage amplifier's output and the signal-to-noise ratio (SNR) are expressed as:

$U_{1}=-\left(I_{i n}+I_{n f 1}\right) \times R_{f 1}=-I_{i n} \times R_{f 1}-I_{n f 1} \times R_{f 1}$,

$\mathrm{SNR}_{0}=\left(I_{\text {in }} \times R_{f 1}\right) /\left(I_{n f 1} \times R_{f 1}\right)=I_{\text {in }} / I_{n f 1}$

The second stage amplifier's output is

$$
\begin{aligned}
& U_{\text {out } 1}=-U_{1} \times \mathrm{R}_{2} / \mathrm{R}_{1}+U_{n 2} \times\left(1+\mathrm{R}_{2} / \mathrm{R}_{1}\right) \\
& =I_{\text {in }} \times R_{f 1} \times \mathrm{R}_{2} / \mathrm{R}_{1}+I_{n f 1} \times R_{f 1} \times \mathrm{R}_{2} / \mathrm{R}_{1}+U_{n 2}\left(1+R_{2} / R_{1}\right) .
\end{aligned}
$$

The signal-to-noise ratio (SNR) of the circuit is expressed as:

$$
\begin{aligned}
& \mathrm{SNR}_{1}=\left(I_{\text {in }} \times R_{f 1} \times \mathrm{R}_{2} / \mathrm{R}_{1}\right) /\left(I_{n f 1} \times R_{f 1} \times \mathrm{R}_{2} / \mathrm{R}_{1}+U_{n 2} \times\left(1+R_{2} / R_{1}\right)\right) \\
& =I_{\text {in }} /\left(I_{n f 1}+U_{n 2} \times\left(1+R_{2} / R_{1}\right) /\left(R_{f 1} \times \mathrm{R}_{2} / \mathrm{R}_{1}\right)\right) .
\end{aligned}
$$

From Equation (3), (4), (5), obviously $\mathrm{SNR}_{1}<\mathrm{SNR}_{0}$. The second stage amplifier expands the output voltage swing, but did not improve the noise performance.

\section{Output voltage swing boost circuit}

Fig. 2(a) shows the modified output swing circuit for ultra-low-noise transimpedance amplifier. The high value feedback resistor $\mathrm{R}_{\mathrm{f} 2}$ is connected between two stages, constitutes a large loop feedback amplifier. The current conversion gain is calculated as $U_{\text {out } 2} / I_{\text {in }}=R_{\mathrm{f} 2}$. The noise voltage brought by $\mathrm{A}_{2}$ is included in the large loop feedback, and are eliminated by feedback.

The output voltage swing is determined by the second stage amplifier $\mathrm{A}_{2}$, which can be extended to more than $\pm 22 \mathrm{~V}$ for most general amplifier. However, an additional loop phase lag may introduced by $\mathrm{A}_{2}$, so a compensation circuit (including $\mathrm{Cc}$ and $\mathrm{Rc}$ ) is involved to provide extra zero point in order to guarantee adequate phase margin.

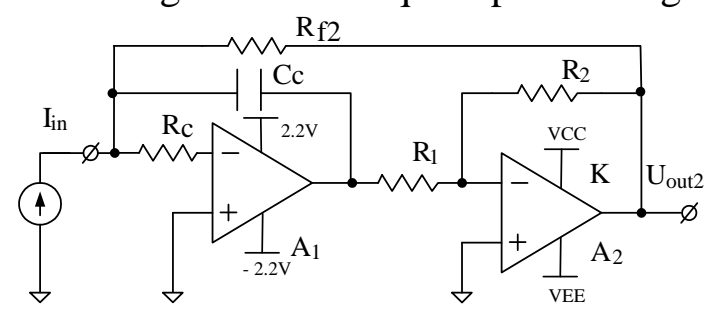

(a)

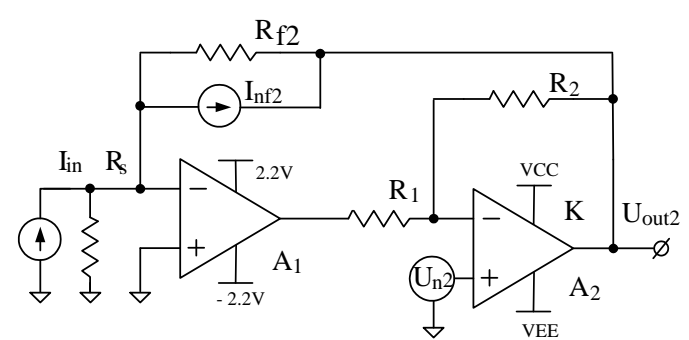

(b)

Figure 2. Output voltage swing boost circuit (a), and its noise equivalent circuit (b).

Fig. 2(b) shows the noise equivalent circuit. Because the output swing of $A_{2}$ is much higher than $\mathrm{A}_{1}$, the feedback resistor $R_{f 2}$ can use a much higher value, and the noise performance will be better. Assume that the gain of second stage is $\mathrm{K}=\mathrm{R} 2 / \mathrm{R} 1$, while its output is $U_{\text {out } 2}=U_{\text {out } 1}$, it leads to $R_{f 2}=$ 
$K \times R_{f 1}$. The equivalent input noise current $\left(I_{n f 2}\right)$ and the signal-to-noise ratio $\left(\mathrm{SNR}_{2}\right)$ of the circuit are expressed as:

$$
\begin{aligned}
& I_{n f 2}=\sqrt{4 k T B / R_{f 2}}=I_{n f 1} / \sqrt{K}, \\
& \mathrm{SNR}_{2}=I_{i n} / I_{n f 2}=\sqrt{K} \times S N R_{0} .
\end{aligned}
$$

Compared to the typical transimpedance amplifier, the feedback resistor $R_{f}$ is much higher, and the noise current and the SNR performance are better. For example: in case of $K=10$, the output swing can be expand to more than $\pm 20 \mathrm{~V}$, meanwhile the SNR will increase to $3.16 \times \mathrm{SNR}_{0}$. Both high voltage output and low-noise performance are achieved.

\section{Performances testing}

Based on the analysis in Section 3, we designed a prototype of improved amplifier with current gain of 20V/200pA. The first stage uses the LMP7721(with typical values of $3 \mathrm{fA}$ input bias, and supply voltage $\pm 2.2 \mathrm{~V}$ ), the second amplifier stage is OP37 (maximum supply voltage of $\pm 22 \mathrm{~V}$ ). The feedback resistor is as high as $100 \mathrm{G} \Omega$, and ideal Johnson noise $0.4 \mathrm{fA} / \sqrt{\mathrm{Hz}}$ at $23^{\circ} \mathrm{C}$.

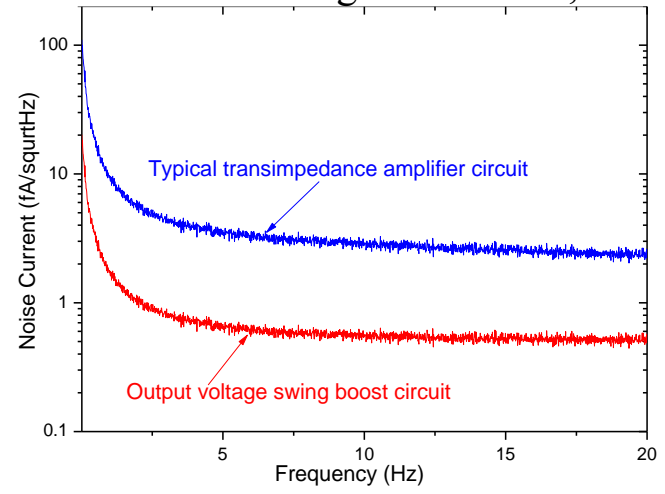

(a)

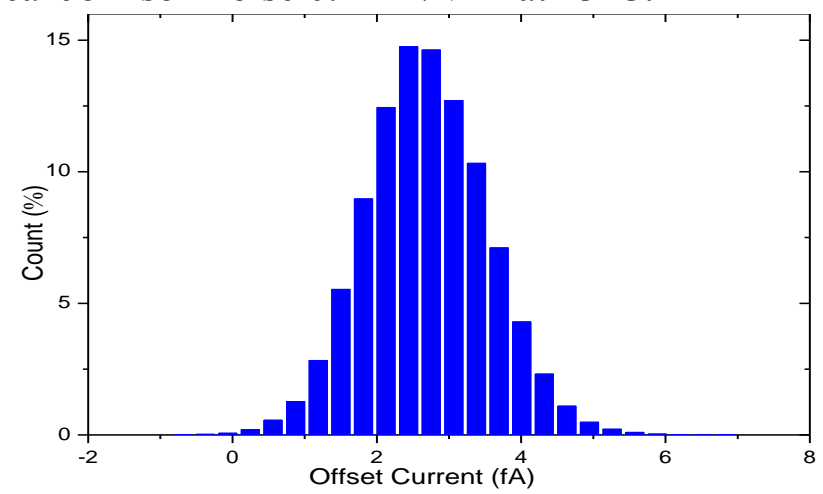

(b)

Figure 3. The noise spectrum (a) and histogram of quiescent bias current (b).

Noise performance was investigated in the frequency range $10 \mathrm{mHz}-20 \mathrm{~Hz}$. Output voltage was recorded by a voltage meter (Keysight DMM-4050) with a sampling rate of $~ 100 \mathrm{sps}$, and then the noise spectra were obtained via Fourier transform. The spectrum of the noise is shown in Fig. 3 (a).

The result indicates that a low white noise level of $\sim 0.5 \mathrm{fA} / \sqrt{\mathrm{Hz}}$ was achieved .Compared to the typical transimpedance amplifier with a feedback resistor of $10 \mathrm{G} \Omega$, the noise current is of $\sim 2.2$ $\mathrm{fA} / \sqrt{\mathrm{Hz}}$, which is nearly a quarter of it. Fig. 3 (b) is a histogram of the input offset current, which exhibits a Gaussian distribution with an average offset of $2.5 \mathrm{fA}$.

The output voltage is also tested, as shown in Table 1. The output voltage is more over the supply voltage of the first stage amplifier, and is also increases linearly with the input current with the relative error no more than $1 \%$.

Table.1 Measurement results

\begin{tabular}{ccccccccc} 
Input Current (pA) & $\mathbf{1 . 0 0 0}$ & $\mathbf{5 . 0 0 0}$ & $\mathbf{1 0 . 0 0}$ & $\mathbf{5 0 . 0 0}$ & $\mathbf{1 0 0 . 0}$ & $\mathbf{1 5 0 . 0}$ & $\mathbf{2 0 0 . 0}$ \\
\hline Measured Voltage (V) & 0.099 & 0.497 & 1.005 & 5.003 & 10.000 & 14.979 & 19.988 \\
\hline Relative Error (\%) & -1 & -0.6 & 0.5 & 0.06 & 0 & -0.02 & -0.06 \\
\hline
\end{tabular}

\section{Summary}

In this paper, an improved low-noise transimpedance amplifier with a large feedback loop is proposed, so that high-value resistor can be employed to achieve low noise performance and wide voltage dynamic range. The proposed amplifier with measurement range of 20V/200pA has been tested: a low white noise level of $0.5 \mathrm{fA} / \sqrt{\mathrm{Hz}}$, and a offset bias of $\sim 2.5 \mathrm{fA}$, while providing an output voltage swing expand to above $\pm 20 \mathrm{~V}$. The proposal can be adopted in many microcurrent applications, 
such as air pollution particles analysis, electrochemical emission supervision, and ionic current measurement of cells.

\section{References}

[1] K. A. Nicoll, Note: A self-calibrating electrometer for atmospheric charge measurements from a balloon platform, Review of Scientific Instruments, 2013, 84(9): 096107.

[2] Carlà M, Lanzi L, Pallecchi E, et al. Development of an ultralow current amplifier for scanning tunneling microscopy. Review of scientific instruments, 2004, 75(2):p. 497-501.

[3] Hassan M, Bushra N, Haq I, et al. Detection and processing of full channel ionic current through indigenized Patch Clamp Technique. 2011 1st Middle East Conference on Biomedical Engineering. IEEE, 2011:p. 386-391.

[4] Kim J, Wang G, Dunbar W B, et al. An integrated patch-clamp amplifier for ultra-low current measurement on solid-state nanopore. SoC Design Conference (ISOCC), 2010 International. IEEE, 2010:p. 424-427.

[5] Zhou C Y, Su H, Mao R S, et al. An accurate low current measurement circuit for heavy iron beam current monitor. Nuclear Instruments and Methods in Physics Research Section B: Beam Interactions with Materials and Atoms, 2012, 280: p.84-87. 